Anthrbio [course]

17 December 2012

\title{
The Human Revolution
}

The evolution of modern humans has long been a keen interest of anthropologists. As a species, humans are interested in knowing why they came to be what they are, today, as well as how they differ from earlier hominins. The anatomical changes that make anatomically modern humans distinctly different from early Homo sapiens - such as changes in skull shape, an increase in cranial capacity, and a decrease in overall robusticity-appeared somewhere between 200 and 100 kya. While fossil and DNA evidence give anthropologists a fairly concrete idea of where and when modern human morphology evolved, the same cannot be said about modern human behavior (Boyd and Silk 303). Anthropologists agree with behavioral shifts associated with the advent of anatomically modern humans, including more efficient foraging techniques, more elaborate tools and complex social organizations, use of symbolic expression, and even the possibility of fully developed language, but not about where and when this "explosion" of modern human culture and behavior began or how it spread (MacLatchy "Homo sapiens"). With more fossil evidence from Africa and increasing support for the Replacement Model or "Out of Africa" theory to explain the dispersal of modern humans, it is now believed that modern behavior evolved gradually along with modern human morphology over a period of 200,000 years as opposed to an abrupt transition during the Upper Paleolithic period.

For a long time, the overriding theory regarding the evolution of behavioral modernity was that some variation of a "human revolution" or "creative explosion" occurred abruptly 
between 40 and 50 kya. The archaeological record provides considerable evidence of modern human behaviors unique from Neanderthals arising almost simultaneously throughout Europe. This evidence pointed to anatomically modern humans being able to inhabit broader ecological ranges, make use of sophisticated tool, construct elaborate shelters, and live within complex social organizations. Evidence of such behavior was also found in Australia and Asia at this time, but, initially, there was very little indication of what was happening in Africa (Boyd and Silk 308). However, recent evidence including resource exploitation in Eritrea and sophisticated tool use in Congo has indicated that modern behavior may have begun in Africa well before its onset in Europe - as far back as 180 kya (MacLatchy "Homo sapiens"). In this way, the extensive European archaeological record contributed toward an early, Eurocentric, and biased conclusion that an "explosion" of modernity occurred in the Upper Paleolithic.

The abrupt transition towards modern human behavior also coincided with the migration of modern people from Africa to Europe $60 \mathrm{kya}$, thus indicating a correlation between the two events. Modern humans' shallow genetic roots, limited interbreeding, and discontinuous Old World skeletal structures provide support for the Replacement Model of modern human dispersal (MacLatchy "Homo sapiens"). Consequently, the fact that this model is so widely accepted has large implications for the human revolution theory. The Replacement Model suggests that Homo sapiens had a single origin in Africa and then spread out and replaced other populations with very little gene flow (MacLatchy "Homo sapiens"). This makes sense regarding the appearance of modern behavior in Africa. Though some argue that this still points toward an abrupt transition in which one key innovation evolved in one human population and spread like wildfire, others believe that the complex behaviors that appeared so abruptly in Europe were actually gradually evolving in Africa between 250 and 60 kya. Thus, the behavioral shift that 
happened during this so-called revolutionary event in Europe was likely just the result of more advanced immigrants from Africa replacing the Neanderthals who resided in Eurasia up until that time (Boyd and Silk 329).

Given that there was no observable major anatomical change in the human species to accompany this "revolution," it becomes difficult to assume that such a huge cognitive transition could have occurred so quickly (MacLatchy "Homo sapiens"). Additionally, evidence concluding that human behavior increased in complexity at about the same time in both Africa and Europe suggests that this transition didn't just occur suddenly in one region, but rather spread out over an extended period of time. Furthermore, the arrival of anatomically modern humans around this same time period explains that, while the transition may have occurred suddenly with the arrival of technologically and culturally advanced African immigrants dominating Neanderthals in Europe, it's possible these behaviors had been gradually evolving for just as long as anatomically modern humans - over 190,000 years. All in all, the human revolution theory seems to have been a premature conclusion for a very gradual behavioral shift toward modernity in human evolutionary history. 